\title{
Chapter I.1
}

\section{Genetic Analysis of Type 1 Diabetes: Embryonic Stem Cells as New Tools to Unlock Biological M echanisms in Type 1 Diabetes}

\author{
Nick Holmes and Anne Cooke \\ Department of Pathology, University of Cambridge, Tennis Court Road, Cambridge CB2 1QP, UK. \\ Address correspondence to: Nick Holmes, e-mail nh106@cam.ac.uk
}

Manuscript submitted December 28, 2012; resubmitted Januar y 9, 2013; accepted Januar y 10, 2013

\section{Abstract}

The nonobese diabetic (NOD) mouse has provided an important animal model for studying the mechanism and genetics of type 1 diabetes over the past 30 years. Arguably, the bio-breeding (BB) rat model may be an even closer phenotypic mimic of the typical human disease. A large number of distinct genetic traits which influence diabetes development have been defined through an extraordinary effort, most conspicuously in the mouse model. However, in both NOD and BB models the lack of availability of robust means for experimental genetic manipulation has restricted our understanding of the mechanisms underlying this spontane-

\section{NOD congenic mice}

敞 $n$ the NOD model, a great deal of effort has . been invested in the genetic dissection of dis- ease susceptibility. This has resulted in the description of more than 35 different chromosomal regions, spread over 14 of the 19 mouse autosomes, which influence the development of type 1 diabetes (T1D) in the mouse $[1,2]$. The majority of these data have been obtained by painstaking effort to produce a large number of congenic mouse strains of the type in which all but one segment of a single chromosome is derived from the NOD mouse. The non-NOD DNA has been introduced by first intercrossing NOD with a mouse strain which does not spontaneously develop diabetes, and then by serial backcrossing selected animals to NOD, normally for 12 or more generations. ous autoimmune disease. Recent developments in the derivation of embryonic stem (ES) cells have the potential to transform this picture. We argue here that targeting of NOD strain ES cells can bring much needed certainty to our present understanding of the genetics of type 1 diabetes in the NOD mouse. In addition, ES cells can play important roles in the future, in both the NOD mouse and BB rat models, through the generation of new tools to investigate the mechanisms by which genetic variation acts to promote diabetes.

Keywords: type 1 diabetes - embryonic stem cell · nonobese diabetic mouse $\cdot$ NOD $\cdot$ gene $\cdot$ susceptibility $\cdot$ haplotype

The limits of the introgressed interval can be defined by typing for markers which vary between NOD and the donor strain. Typically, despite the heroic efforts of the investigators, these intervals are several megabases in size and contain several, sometimes dozens, of annotated genes [2]. In many cases, the degree of genetic diversity between the NOD strain and introgressed donor DNA is not fully known. While the C57BL/6 (B6) reference genome is essentially complete and extensive sequence data for NOD exists, annotated sequence coverage of the NOD genome in publically available databases is currently limited. F urthermore, $\mathrm{B} 6$ is the congenic donor only in certain strains; C57BL/10SnJ (B10), nonobese nondiabetic (NON), nonobese-resistant (NOR) and, less frequently, many other strains have been used to produce NOD congenics [1]. While B10 has many fewer dif- 


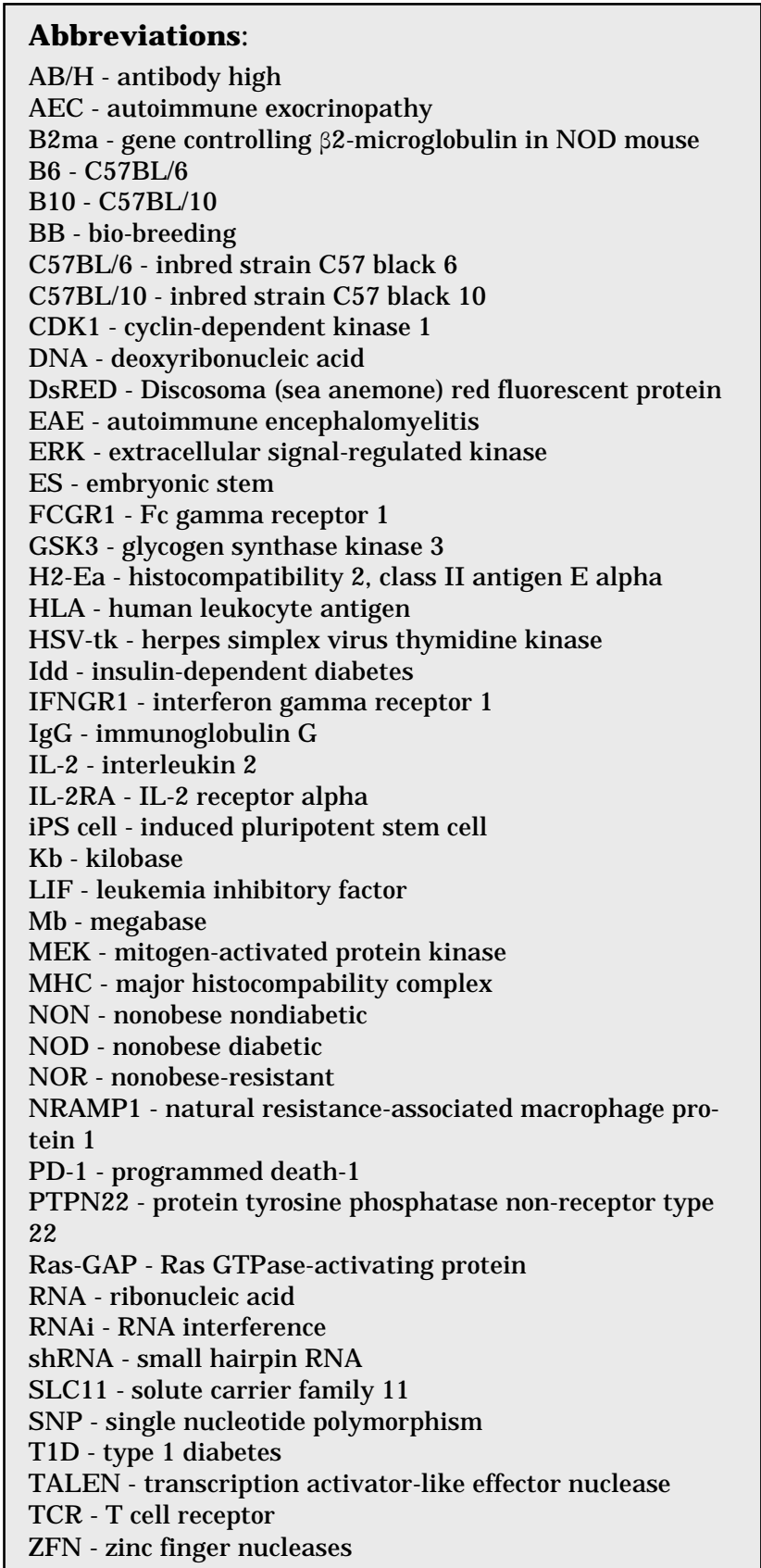

ferences with B6 than NOD [3], these are not all quantified or mapped. There is considerable variation in the degree of sequence diversity between B6 and NOD, but in some places it is high ( $1 \%)$ [4]. This has helped the precision with which the recombination boundaries of the susceptibility intervals can be defined. However, it means that even regions of $2 \mathrm{Mb}$ typically contain more than a few hundred differences [2] which renders attributing causation to any individual sequence variant impossible.
With the completion of the mouse genome project, it is certainly possible to inspect the susceptibility regions for annotated genes and use knowledge of these genes and their products to make informed guesses about possible candidates. Obviously, in some cases this is easier than in others, but in all cases there must be a degree of uncertainty about the identity of the causative gene and even more so about the identity of the variant or variants which lead to the susceptibility phenotype. It is certainly true that even with a good deal of circumstantial evidence in their favor, hypothetical identification of causative genes may finger the wrong candidate. A good example of this is the Idd3 region on chromosome 3 (see next section).

The study of congenic strains selected for re duced diabetes incidence has been complemented by at least three distinct approaches to probing candidate genes.

\section{Correlating genetic, phenotypic, and functional variation}

If congenic mouse strains have identified and partially localized a susceptibility/resistance polymorphic trait, an obvious method to identify candidate genes is to examine their known function and to look for specific allelic variation correlating with some functional phenotype which might plausibly be involved in the development of T1D. While the current state of all Idd regions includes multiple genes, some contain deliciously tempting possibilities whose known biology positively invites hypothetical imputation. However, inevitably 'cherry picking' of what appear likely candidates suffers from bias imposed by our ignorance and limited by our imaginations; there is surely a case that imputations have been too immunologically focused and that genes affecting beta-cell intrinsic functions have been too little considered [5].

The first, and still most significant, susceptibility region described was Iddl. This region contains the MHC. It was established early on that the MHC of NOD mice contained an haplotype of linked MHC alleles, shared only with the closely related nonobese-resistant (NOR) strain [6]. Within that haplotype was a single functional $\mathrm{MHC}$ class II gene pair with the $\mathrm{H} 2-\mathrm{Ab}^{97}$ allele found only in NOD, NOR, and Biozzi AB/H strains [7]. This class II beta chain had two particularly distinctive amino acids at positions 56 and 57 (HisSer) [6]; position 57 was considered especially significant since human HLA-DQbeta alleles asso- 
ciated with T1D also lacked the Asp residue found at this position in the majority of alleles associated with resistance [8]. Of course, the known function of $\mathrm{H}-2 \mathrm{~A}$ was also a strongly supportive factor; polymorphism at this locus affecting peptide selection for antigen presentation, shaping the TCR repertoire, and thus potentially influencing both central and peripheral tolerance. In this case, transgenic mice made on the NOD background showed that additional expression of $\mathrm{H}^{-2} \mathrm{Abl}^{97}$ genes mutated to express the consensus residue at either position 56 (Pro) or 57 (Asp), either abolished (Pro56) [9] or significantly reduced (Asp57) [10] the incidence of T1D despite the continued expression of the native $\mathrm{H}-2 \mathrm{Ab} 1^{{ }^{77}}$ allele. Furthermore, it was shown that diabetes onset was de pendent on the lack of a functional H2-Ea gene [9]. Even the most cautious commentator would have to say that we are as sure as possible that the $\mathrm{H}$ 2Abl locus is an important T1D susceptibility locus, and that the 5 nucleotide polymorphisms responsible for the double amino acid change are in all likelihood, at least in part, causative. We can have equal confidence that the 629bp deletion in the $\mathrm{H} 2-\mathrm{Ea}$ gene is al so a cause of T1D susceptibility. What we cannot say, however, is that there are no other variants within the NOD MHC significantly affecting diabetes incidence.

The attribution of causality to the $\mathrm{H}-2 \mathrm{Abl}$ gene may be correct, but the identification of causative genes and variants within other chromosomal intervals associated with susceptibility or resistance to diabetes has proved fraught with difficulty. This is well illustrated by the history of the Idd3 region. Idd3 was originally described as lying within a large segment of chromosome 3 [11]. However, the disease-associated variants between B6 and NOD on chromosome 3 are now believed to contain no less than six traits that contribute to susceptibility (renamed Idd3, 17, 10, 18.1, 18.3 and 18.4) in NOD mice [2]. Before this dissolution, the gene for the high affinity receptor for IgG (F cgr 1 ) was the first to be proposed as responsible for the disease linkage [12]. There were sound arguments advanced for this choice. A functional polymorphism was identified, and the minor allele was present in only NOD and another autoimmune-prone strain, Biozzi AB/H (of 22 strains screened). The polymorphism in Fcgr 1 was extensive, with 24 nucleotide changes, 19 of them non-synonymous, and resulted in a severe truncation of the cytoplasmic tail of the receptor. The variants affected recycling and/or binding of IgG2a molecules to macrophages. Macrophages were already known to be important in T1D both in NOD and in humans. Nevertheless, subsequent work showed that all susceptibility traits could be separated from the F cgrl locus [13].

Following the separation of the chromosome 3 diabetes susceptibility traits into 3 distinct linkage regions, a second plausible candidate was advanced. The now smaller Idd3 region contained the gene for the cytokine IL-2. The size of the potential region was progressively reduced to $780 \mathrm{~Kb}$ which still contained IL-2 [14]. A number of significant polymorphisms between NOD and B6 mice were found in the 112 gene; some were upstream of the gene, while others altered the $\mathrm{N}$ terminal sequence and subsequent glycosylation of the protein. I L-2 was known to be involved in regulating autoimmunity, and IL2RA was a candidate gene in human T1D susceptibility. This led Lyons et al. to examine IL-2 expression levels in Idd3 discordant mice. They found no differences and concluded that the protein differences in IL-2 were most likely responsible for Idd3 [14]. However, a later study by an overlapping group of authors concluded that there were transcriptional differences between strains with I dd3 from different genetic origins, and that the lower level of IL-2 transcription was concordant with susceptibility, whereas protein-altering variants did not correlate in the same comparison [4]. They went on to show that NOD background mice which were heterozygous for an Idd3-containing interval from a 129derived II 2 knockout had both altered IL-2 levels and disease susceptibility. This later study therefore made the opposite conclusion to the 2000 report, namely that differences in IL-2 expression and not protein-encoding changes were responsible for the I dd 3 disease affect.

Nevertheless, while the Yamanouchi study presented a strong argument for polymorphisms upstream of 112 being responsible, there remains some uncertainty. The discrepancy between the Lyons and the Yamanouchi studies regarding the correlation of IL-2 levels with disease susceptibility illustrates the difficulties in being certain what cell type and/or activation state is the critical population in which to measure expression. The II21 gene lies within about $100 \mathrm{~Kb}$ of $\mathrm{II} 2$ and inside the Idd3 region. There is also compelling evidence that IL-21 to IL-21R interaction is important in T1D [15 -18]. The II 2 knockout was made by placing an active neo gene within the third exon of 112 [19]; to our knowledge no studies have been conducted to show that it does not alter IL-21 expression. Expression of the neo gene is expected to be constitutive and strong, driven by a hybrid HSV-tk promoter and a tandem repeat of the enhancer region from the polyoma mutant PYF441 [20]. Ef- 
fects on the transcription of neighboring genes have been found in this type of knockout, some at quite long range [21].

\section{Introduction of targeted mutations from other genetic backgrounds}

A large number of knockout alleles have been backcrossed onto the NOD background; the degree to which these NOD knockouts have been shown to be genetically identical to parental NOD at unlinked loci and the genetic interval to which re combination has reduced the introgressed B6 or 129 DNA is variable. Frequently, up to 12 generations of backcross have been employed and sometimes microsatellite typing has been used to check for adventitious unlinked non-NOD DNA. In many cases the knockout reduced or eliminated the incidence of diabetes. However, in a minority of cases the knockout (e.g. of PD-1 [22]) has exacerbated disease.

While it is obviously attractive to take advantage of the availability of the rich genetic resource of targeted mutations made on the 129 and B6 backgrounds, the problems of backcrossing require patience, thorough characterization and careful interpretation. In all cases, there will be a significant segment of chromosome surrounding the induced mutation which is derived from the original targeted strain even after 12 generations of backcross; the extent will depend on the distribution of recombination within the relevant genomic region. There may also be other adventitious segments of chromosome inherited from the ES cells or later strains used. Even where the manipulated allele does not lie close to any known diabetes susceptibility/resistance allelic variation, but the targeted allele derives from 129, there may be confounding effects of linked alleles, since most of the known Idd regions have been defined only by variation between NOD and strains other than 129 (mostly B10, B6, NON and NOR). An early example of this problem was provided by the interferon gamma receptor, the knockout of which initially appeared to prevent diabetes in mouse lines backcrossed 10 or 11 generations to NOD. In fact, a single line established at N14 showed that the resistant phenotype was due to a linked gene [23]. No known Idd re gions lie on chromosome 10 (where the I fngr 1 gene is found), and the 129 allelic variant responsible for diabetes resistance has not been identified. It should be borne in mind that this problem of confounding effects of linked genes is a general one in mouse knockout studies where the knockout strain has to be backcrossed for functional studies. Studies have shown that the ES-linked region retained is typically $25-40 \mathrm{Mb}$ and that a significant proportion of genes within this congenic segment displays altered transcription levels [24, 25]. Furthermore, sometimes the ES-cell passenger alleles may accelerate diabetes since resistance variants are also present in NOD [26, 27].

The use of knockout alleles originally made in 129 ES cells has, however, been fruitful when combined with transgene rescue. This approach enabled Slattery and colleagues to provide very convincing evidence that beta-2 microglobulin was a diabetes susceptibility locus and probably responsible for the Idd13 resistance region (derived from the NOR strain). While backcross of a beta-2 microglobulin knockout allele from a 129 ES cell targeting event blocked diabetes completely, as might be expected given the effects on development of CD8 T cells, Slattery et al. showed that complementation with a cDNA transgene encoding the $B 2 \mathrm{~m}^{\mathrm{a}}$ allotype (identical to the NOD form) restored the disease incidence, whereas the $\mathrm{B} 2 \mathrm{~m}^{\mathrm{b}}$ allele (identical to NOR) did not [28]. Both transgenes restored $\mathrm{MHC}$ class I protein expression and CD8 development, suggesting a subtle effect on the interaction of MHC class I molecules with peptide and/or their multiple receptors.

\section{RNA interference approaches}

Kissler has pioneered the use of lentiviral shRNA transgenics as a strategy to investigate the effects of knockdown of specific gene products in the NOD background. This approach has some distinct advantages. First, the transgenic mice can be produced by introducing the foreign DNA directly into the canonical NOD strain, thus avoiding all the problems of inheritance of linked and unlinked alleles from other strains inherent in the backcross approach. Second, in later versions of the lentiviral vector, the expression of the shRNA can be made inducible and reversible by means of an $\mathrm{H}$-tetO promoter. Drawbacks associated with this approach are (a) that knockdown of the target is often incomplete (b) there may be off-target effects of the shRNA and (c) there may be position effects from the inserted DNA. These disadvantages could be mitigated by the use of multiple shRNA sequences and independent transgenic lines with different insertion sites. Kissler and collaborators used this approach to test the hypothesis that the gene SIcllal (NRAMP1) is responsible for the susceptibility associated with the Idd5. 2 genetic in- 
terval, a gene-rich region of $1.48 \mathrm{Mb}$ containing 48 annotated protein encoding genes. Their data strongly supported the notion that the B10 allele at SIcllal is responsible for reduction in diabetes incidence in NOD.B10Sn-Idd5.2 congenic mice [29]. This conclusion was assisted by the fact that the B10 allele is believed to encode a null-function protein, despite differing from the functional $A / J$ strain allele by a single amino acid only (position 169), thus rendering the knockdown a near exact phenocopy of the B10 allele. Clearly this will not be the case with most allelic variants.

Perhaps because of this limitation, to date no other NOD susceptibility candidates have been interrogated using RNAi; instead two candidates for human diabetes susceptibility traits have been investigated in the NOD model, variation in soluble CTLA4 expression and an amino acid variant of the phosphatase PTPN22. These knockdowns had opposite effects on disease; partial silencing of sCTLA4 increased disease in NOD.B10Sn-Idd5.1 congenics, though not the parental NOD background [30], and a reduction of $80 \%$ in PTPN22 expression reduced diabetes onset in NOD transgenics [31].

\section{The development of ES cells from NOD mice}

The development of pluripotent mouse cells capable of both genetic modification during in vitro culture and generating novel 'designer' animals has revolutionized mouse genetics. Later, it be came apparent that there were potent genetic effects on the ability to isolate such germline competent embryonic stem (ES) cells. This means that only a few strains of mice could be successfully used to produce such cells by the canonical culture method; most strains, including NOD, are said to be "refractory" with respect to pluripotent ES cell derivation. The first description of the isoIation of ES cells from NOD mice, indeed from any spontaneous autoimmune disease model, was provided by Nagafuchi and colleagues in 1999 [32]. They produced 5 ES cell lines by a classical method involving culture of blastocyst-derived cells on primary mouse embryonic fibroblast feeders in the presence of leukemia inhibitory factor (LIF). Only one of these lines retained both ES-like morphology and normal karyotype during serial passage. This ES line was injected into C57BL/6 blastocysts which were reimplanted into pseudopregnant ICR females to produce 22 chimeras ranging from $30 \%$ to over $80 \% \mathrm{ES}$ (NOD) contribution by coat color. However, chimerism, as meas- ured by peripheral blood lymphocyte $\mathrm{H}-2$ allotype, was much lower $(\varangle 0.2 \%)$. Nevertheless, germline transmission was obtained, but only at very low efficiency (1/97 offspring were NOD ES-derived) [32].

Arai and colleagues subsequently made significant improvements to the effectiveness of this first NOD ES line by altering culture conditions. They found that NOD ES cells required a 10-times higher concentration of LIF to prevent differentiation than did 129 (E14.1 and D3) ES lines, and that distinct batches of FCS were optimal for survival and undifferentiated appearance of NOD ES versus 129 ES cells [33]. Most critically, they showed that injection into NOD rather than B6 blastocysts generated chimeras with much higher germline transmission (25-60\%). Practically however, making chimeras in NOD blastocysts would be a severe limitation since the screening of chimerism and transmission can only be done by detecting genetic markers introduced into the ES cells. Chen et al. provided a possible solution to this problem by deriving a congenic NOD mouse with an agouti coat. They also isolated their own NOD ES cells. However, while these cells produced chimeras with the NOD.CBALs-Tyr ${ }^{+} /$Lt blastocysts, they did not transmit through the germline [34]. We are not aware of any targeted mutant NOD mice having been produced by ES cells from either of these studies.

Brook et al. next reported an unsuccessful attempt to derive NOD strain ES cells using microsurgery to explant epiblasts of blastocysts which had been subjected to implantation delay initiated by ovariectomization [35], a technique which they had successfully applied to another refractory strain, CBA/Ca. In the case of NOD mice, few viable blastocysts were recovered; transfer of NOD conceptuses to non-NOD dams produced 9 lines, but all differentiated during passage. They were similarly unsuccessful with NOD congenics which do not develop diabetes. However, when (NOD $\times$ 129)F 1 embryos were used, the success rate in ES derivation was $-83 \%$, and the derived ES cells made germline competent chimeras at high efficiency [35]. Brook et al. also found that novel congenic strains, made by backcrossing the (NOD $\times 129) F 1$ to 129 and subsequent intercross, also made ES cells (at generation N2F4). In one case, these were shown to have good germline competence.

After several years of unsuccessful endeavor, four breakthrough papers describing NOD ES cells appeared in 2009. A critical advance which enabled germline-competent NOD background ES 
cells to be isolated with high efficiency was made by the discovery in 2008 that ES cells could be maintained in a pluripotent state without LIF or serum by culture in " $3 \mathrm{i}$ medium" containing 3 small molecule enzyme inhibitors (SU5402 for FGFR, PD184352 for ERK, and CHIR99021 for GSK3) [36]. Ohta et al. used 3i medium to isolate several lines of ES cells from NOD mice [37]. These ES cells were only moderately efficient (2.5$10 \%$ ) in generating chimeras, but the chimeras themselves gave germline transmission at high frequency (42-100\%). Yang et al. used a different inhibitor (SC-1, i.e. pluripotin, which inhibits both ERK 1 and Ras-GAP) in combination with LIF to isolate four ES cell lines from NOD-scid mice [38]. It was noticeable that the efficiency with which NOD-scid ES lines produced chimeras was significantly lower than that of other refractory lines (SCID-beige, CD-1). The single successful line gave only 1 chimera from 35 pups born. No data were provided for germline transmission.

Two other independent studies derived more efficient NOD ES cell lines. Hanna et al. used the following inhibitors: (i) Kenpaullone alone, (ii) Kenpaullone in combination with CHIR99021, or (iii) the more typical $2 \mathrm{i}$ system (PD184352 + CHIR99021) [39]. Kenpaullone inhibits both GSK $3 \beta$ and CDK1/cyclin B. All three conditions produced NOD ES cells. A single line from each condition was shown to give germline chimeras. Nichols et al. employed yet another $2 \mathrm{i}$ system (PD0325901, an MEK inhibitor, and CHIR99021), with LIF to derive NOD ES lines with $53 \%$ efficiency. This was considerably higher than that achieved in the conditions used by Hanna et al.; four of these lines were tested by blastocyst injection, and all gave high rates of chimerism (100$60 \%$ ) and germline transmission (100-75\%) [40].

\section{Genetic manipulation of NOD ES cells}

Obviously, aside from the intrinsic interest in understanding the determinants of embryonic cell pluripotency and differentiation, one of the principal reasons for deriving NOD ES cells is to permit genetic manipulation of NOD mice to further our understanding of the genetics and mechanism of T1D. Thus, it was important to demonstrate that NOD ES cells could be modified, and that mice de rived from $E S$ cells would develop diabetes at the expected rate unless intended modifications altered relevant properties. Nichols et al. showed that one NOD ES cell line could be stably trans- fected with a DSRED expression cassette using PiggyBac transposition, selected by culture in hygromycin, and still give efficient germline competent chimeras [40]. Hanna et al. demonstrated that the Nanog gene could be targeted by homologous recombination in one NOD ES line, but did not re port testing chimeric or germline potential after targeting [39]. Nichols et al. also showed that mice derived from unmanipulated NOD ES cells have the same rate of onset and diabetes incidence as 'standard' NOD mice from the same colony [40]. Formally, the incidence cohort of mice, the progeny of NOD-ES $\rightarrow$ C57BL/6 chimeric males mated with NOD females, was $50 \%$ ES-derived. This latter point is of great importance since ES cells in culture may undergo adventitious genetic and epigenetic changes which have the potential to alter diabetes incidence in addition to restricting their germline potential.

There is one very recent publication, by Morgan et al., describing the derivation of genetically targeted NOD mice from pure NOD ES cells [41]. This study shows that the targeted disruption of the $\mathrm{H}-2 \mathrm{DM}$ a gene in NOD mice prevents diabetes onset. Prior to this, there was a report of the derivation of knockin mice from (NOD $\times 129$ )F $1 \mathrm{ES}$ cells [42]. Kamanaka et al. used homologous targeting to replace exons 1 and 2 (plus intron 1 and part of intron 2) of the 112 gene with the equivalent B6 genomic DNA. The F1 ES cells were generated by classical means and gave high efficiency chimerism and germline transmission. Ten targeted clones were produced, 3 of which were used to generate subclones in which the inserted floxed neo gene had been removed. All 3 gave rise to germline competent chimeras. However, the use of (NOD $\times$ 129)F1 ES cells necessitated two further steps. First, it was essential to determine which parental chromosome in the ES cells had been targeted. This had to be done after breeding the chimeras because of the high degree of local homology between 129 and NOD. A flanking microsatellite showed that two clones had targeted the 129 allele and one the NOD allele. Second, a laborious backcross had to be undertaken to render the genome homozygous for NOD DNA with the chromosome 3 segment containing the targeted II 2 gene. Despite the use of a "speed-backcross" approach, selecting at each generation for the greatest number of NOD alleles at Idd markers, 12 generations were required. The results confirmed that the sequence variation between NOD and B6 at the $\mathrm{N}$-terminus of the IL-2 protein does not alter the incidence of T1D in NOD background mice and cannot be re- 
sponsible for the B6-Idd3 resistance effect [42], in agreement with the earlier proposal by Yamanouchi et al. [4].

\section{The future potential of ES cells and alternative targeting approaches in type 1 diabetes}

We will ignore here the potentially important role that both mouse and human ES and iPS cells are likely to play in the development of beta-cell replacement therapies since this topic has been reviewed elsewhere [43]. I nstead, we focus on the potential for the exploration of the genetic and mechanistic basis of T1D which is unlocked by targeted germline manipulation. Given the biological resources generated by Hanna and Nichols [39, 40], the NOD mouse field is clearly the most likely to benefit. However, the inhibitor culture approach has also been shown to work for deriving rat ES cells, and it is to be hoped that this will be used on the $B B$ rat model of diabetes $[39,44]$. In addition, there has been significant recent progress in genetic modification of rats (and many other diverse organisms) using 'designer nucleases' intended to cleave at unique sites within the genome $[45,46]$.

Zinc finger nucleases (ZFN) and transcription activator-like effector nucleases (TALENs) represent an alternative approach to gene targeting. This approach is not limited to use in cells with intrinsically high homologous recombination efficiency such as ES cells and can be deployed by direct microinjection of mRNA and/or DNA into zygotes [47-49]. Such designer nucleases have potential for modification of ES cells, including NOD ES cells, and for directly targeting the genome of BB rats and NOD mice. The extent to which this new technology replaces ES cells will depend on the precise type of modification sought and the extent to which problems associated with the use of nuclease, particularly the generation of small deletions or insertions at both the targeted site and at off-target sites elsewhere in the genome can be overcome [50].

We would suggest that exploitation of BB rat germline modification might focus on three avenues where the rat model offers advantages over the mouse:

1. Genetic regions where there may be susceptibility traits common between rats and humans which are absent in mouse (e.g. rat Iddm34 [2])
2. Putative biological pathways which are more experimentally accessible in the larger rodent

3. Studies specifically aimed at understanding those complications of diabetes, e.g. nephropathy, which are better mirrored in the rat model [51]

Furthermore, both NOD mice and BB rats can be used to study other autoimmune pathologies; autoimmune thyroid disease (both models) [52, 53], autoimmune exocrinopathy (AEC; a Sjögren's syndrome-like disease in NOD) [54], and they can be induced to develop autoimmune encephalomyelitis (EAE, NOD) [55] and collagen-induced arthritis (BB rat) [56]. Some genetic susceptibility loci are shared by these diseases. However, other strains may constitute better models, either NODrelated e.g. C57BL/6.NOD-Aec1Aec2 [57] for Sjögren's syndrome, or not directly NOD-related, e.g. Biozzi $A B / H$ for $E A E$ [58]. The 2i culture methodology could be applied to the development of ES cell resources from such autoimmune strains to enable the dissection of these models.

For the NOD mouse, there are three rather obvious directions to be followed (summarized in Figure 1). The first, quickest, and simplest strategic direction will be to use targeted modification of NOD ES cells to confirm or disprove the identity of loci for which we already have good evidence. The genes II2 (Idd3), SIclla1 (Idd5.2), Ctla4 (Idd5.1), and Vav3 (Idd18.1) come immediately to mind. In these cases, there is strong evidence, but almost all of it is circumstantial. We are seriously concerned that some authors have confused phenotypic-genotypic correlations, even ones backed up by teleological arguments based on known functions, however plausible, with formal direct tests of hypothetical attribution of diabetes susceptibility genes or even of causative variants. As we discussed above, identification of Idd3 has batted backwards and forwards over time, with protein sequence and transcription levels of IL-2 both favored at different times. Currently the data strongly favor polymorphic variation upstream of the II 2 gene as the causative variants for Idd3. Indeed, del Rio et al. have shown that 6 of the 7 SNP/Indel within the first 1015bp upstream independently influence transcription levels in a reporter assay (albeit one SNP in the opposite direction, NOD>B6) [59]. However, it is quite clear that we cannot say which set of variants between B6 and NOD are responsible for the apparent differences in 112 transcription rates nor, which is rather more important, can we say for certain that 
this phenotype is fully re sponsible for the protection against diabetes offered by B6-Idd3 on the NOD background. We can determine both of these questions with one or more novel targeted NOD strains from NOD ES cells with knockin mutations. A similar argument can be made for all the Idd for which there are good candidate genes (and in the case of Ctla4 and SIcllal, candidate variants). Circumstantial evidence, however strong, is not a substitute for proper critical tests.

The second logical use of NOD ES cells will be to focus down the susceptibility/resistance trait in those Idd regions which are still large and which do not have good candidate genes (see Figure 1). This will require a greater number of targeted ES cells with larger regions 'swapped' for test strain homologous segments and will be more demanding therefore both technically and in resource terms. Perhaps a sensible place to start is to use BAC clones and recombineering technology [60] to replace regions within these large Idd with the relevant re sistant strain over those areas which have human orthologous-associated regions (e.g. Idd2). These might then provide both pinpointing information and mechanistic models.

This brings us to our third potentially valuable use for NOD ES cells, which is to probe mechanisms of susceptibility/resistance both for mouse-specificassociated genes and to generate models of human disease-associated genes. Even where we have faith that we have identified a genuine disease susceptibility gene, our current understanding of mechanism is weak. If we examine the richness that targeted, especially knockin and conditional knockout, mice have provided in exploring immunity in the broader context, the value that single gene or single causative variant knockin transgenic NOD mice could bring to mechanistic studies of T1D development must be apparent. Furthermore, there is clearly scope for the use of reporter mice on the NOD background, both in mechanistic 
studies and in setting up models for developing beta-cell replacement therapies. It is worth re membering that the same $2 \mathrm{i}$ methodology used to derive NOD ES cells with good efficiency can also be applied to NOD transgenic mice derived from single or multiple targeted ES cells (see Figure 1).

There are clearly some in the diabetes field who question whether any new useful information can be obtained from the NOD mouse model. We be lieve that there are further valuable lessons to be learned from studying T1D in NOD. Detailed studies aimed at uncovering the exact polymorphisms responsible for genetic susceptibility and resistance to T1D can be addressed through targeted manipulation and in vivo and ex vivo studies of the

\section{References}

1. Leiter EH. N onobese diabetic mice and the genetics of diabetes susceptibility. C urr D iab R ep 2005. 5:141-148.

2. Burren OS, Adlem EC, Achuthan P, Christensen M, Coulson R M, Todd JA. T 1D Base: update 2011, organization and presentation of large-scale data sets for type 1 diabetes research. N udeic A cids R es 2011. 39:D 997-D 1001.

3. Cervino AC, Gosink M, Fallahi M, Pascal B, Mader C, T sinoremas NF. A comprehensive mouse IBD database for the efficient localization of quantitative trait loci. M amm $\mathrm{G}$ enome 2006. 17:565-574.

4. Yamanouchi J, Rainbow D, Serra P, Howlett S, Hunter K, Garner VE, Gonzalez-Munoz A, Clark J, Veijola R, C ubbon R, et al. Interleukin-2 gene variation impairs regulatory $T$ cell function and causes autoimmunity. $\mathrm{N}$ at $\mathrm{G}$ enet 2007. 39:329-337.

5. Eizirik DL, Sammeth M, Bouckenooghe T, Bottu G, Sisino G, Igoillo-Esteve M, Ortis F, Santin I, Colli $\mathbf{M L}$, Barthson J, et al. The human pancreatic islet transcriptome: expression of candidate genes for type 1 diabetes and the impact of pro-inflammatory cytokines. PL OS G enet 2012. 8:e1002552.

6. Acha-O rbea H, McD evitt HO. The first external domain of the nonobese diabetic mouse class II I-Abeta chain is unique. P roc N atl A cad Sci U SA 1987. 84:2435-2439.

7. Liu GY, Baker D, Fairchild S, Figueroa F, Q uarteyPapafio R, Tone M, Healey D, Cooke A, Turk JL, Wraith DC. Complete characterization of the expressed immune response genes in $B$ iozzi $A B / H$ mice: structural and functional identity between $A B / H$ and $N O D A$ region molecules. Immunogenetics 1993. 37:296-300.

8. Todd JA, Acha-Orbea H, Bell JI, Chao N, Fronek Z, Jacob CO, MCD ermott M, Sinha AA, Timmerman $L$, Steinman L, et al. A molecular basis for M HC class IIassociated autoimmunity. Science 1988. 240:1003-1009.

9. Lund $\mathbf{T}, \mathbf{O}^{\prime}$ Reilly $L$, Hutchings $\mathbf{P}$, Kanagawa $\mathbf{O}$, Simpson E, Gravely R, Chandler P, Dyson J, Picard JK, Edwards A, et al. Prevention of insulin-dependent diabetes mellitus in non-obese diabetic mice by transgenes encoding modified I-A beta-chain or normal I-E alphachain. $N$ ature 1990. 345:727-729.

10. O'Reilly LA, Healey D, Simpson E, Chandler P, Lund T, Ritter MA, Cooke A. Studies on the thymus of non- obese diabetic (N O D) mice: effect of transgene expres- mouse system. Perhaps more importantly the biological pathways influenced by the susceptibility locus can be investigated and validated in murine models in ways which cannot ethically or affordably be conducted in human subjects. NOD ES cells can not only seal the deal on identification of causative genetic variants, but also permit the development of efficient models for development of candidate therapies.

Acknowledgments: We thank Dr J ennifer Nichols for comments on the manuscript. AC acknowledges financial support from the Wellcome Trust.

Disclosure: The authors report no conflict of interests. sion. Immunology 1994. 82:275-286

11. Todd JA, Aitman TJ, Cornall RJ, Ghosh S, Hall JR, Hearne CM, Knight AM, Love JM, McAleer MA, Prins JB, et al. G enetic analysis of autoimmune type 1 diabetes mellitus in mice. $N$ ature 1991. 351:542-547.

12. Prins JB, T odd JA, R odrigues NR, Ghosh S, Hogarth PM, Wicker LS, Gaffney E, Podolin PL, Fischer PA, Sirotina A, Peterson LB. Linkage on chromosome 3 of autoimmune diabetes and defective Fc receptor for IgG in N O D mice. Science 1993. 260:695-698.

13. Podolin PL, Denny P, Lord CJ, Hill NJ, Todd JA, Peterson LB, Wicker LS, Lyons PA. Congenic mapping of the insulin-dependent diabetes (Idd) gene, Idd10, localizes two genes mediating the Idd10 effect and eliminates the candidate Fcgr1. I I mmunol 1997. 159:1835-1843.

14. Lyons PA, Armitage N, Argentina F, Denny P, Hill NJ, Lord CJ, Wilusz MB, Peterson LB, Wicker LS, Todd JA. C ongenic mapping of the type 1 diabetes locus, Idd3, to a 780-kb region of mouse chromosome 3: identification of a candidate segment of ancestral DNA by haplotype mapping. $G$ enome $R$ es 2000. 10:446-453.

15. Spolski R, Kashyap M, Robinson C, Y u Z, Leonard WJ. IL-21 signaling is critical for the development of type I diabetes in the NOD mouse. Proc N atl A cad Sci U SA 2008. 105:14028-14033.

16. Sutherland AP, Van Belle T, Wurster AL, Suto A, Michaud $M$, Z hang D, Grusby $M J$, von Herrath $M$. Interleukin-21 is required for the development of type 1 diabetes in N O D mice. D iabetes 2009. 58:1144-1155.

17. McGuire HM, Walters $S$, Vogelzang $A$, Lee $C M$, Webster KE, Sprent J, Christ D, Grey S, King C. Interleukin- 21 is critically required in autoimmune and allogeneic responses to islet tissue in murine models. D iabetes 2011. 60:867-875.

18. Van Belle $\mathbf{T} L$, Nierkens $\mathbf{S}$, Arens $\mathbf{R}$, von Herrath MG. Interleukin-21 receptor-mediated signals control autoreactive $\mathrm{T}$ cell infiltration in pancreatic islets. Immunity 2012. 36:1060-1072.

19. Schorle H, Holtschke T, Hünig T, Schimpl A, Horak I. D evelopment and function of $T$ cells in mice rendered interleukin-2 deficient by gene targeting. $\mathrm{N}$ ature 1991. 352:621-624.

20. Thomas KR, Capecchi MR. Site-directed mutagenesis by gene-targeting in mouse embryo-derived stem cells. C ell 1987. 51:505-512. 
21. Malissen M, Gillet A, Ardouin L, Bouvier G, Trucy J, Ferrier $\mathbf{P}$, Vivier E, Malissen B. A ltered T cell development in mice with a targeted mutation of the CD3-epsilon gene. EM B O J 1995. 14:4641-4653.

22. Wang J, Yoshida T, Nakaki F, Hiai H, Okazaki T, Honjo T. Establishment of N O D-Pdcd1-/ - mice as an efficient animal model of type I diabetes. Proc N atl Acad Sci USA 2005. 102:11823-11828.

23. Kanagawa O, Xu G, Tevaarwerk A, Vaupel BA. Protection of nonobese diabetic mice from diabetes by gene(s) closely linked to IFN-g receptor loci. J Immunol 2000. 164:3919-3923.

24. Schalkwyk LC, Fernandes C, Nash MW, Kurrikoff K, Vasar E, Koks S. Interpretation of knockout experiments: the congenic footprint. $G$ enes B rain B ehav 2007. 6:299-303.

25. Valor LM, Grant SG. Clustered gene expression changes flank targeted gene loci in knockout mice. PL OS O ne 2007. 2:el303.

26. McAleer MA, Reifsnyder P, Palmer SM, Prochazka $M$, Love JM, Copeman JB, Powell EE, Rodrigues NR, Prins JB, Serreze DV, et al. C rosses of N OD mice with the related N ON strain. A polygenic model for IDDM. D iabetes 1995. 44:1186-1195.

27. Gonzalez A, Katz JD, Mattei MG, Kikutani H, Benoist C, Mathis D. Genetic control of diabetes progression. Immunity 1997. 7:873-883.

28. Hamilton-Williams EH, Serreze DV, Charlton B, Johnson EA, Marron MP, Mullbacher A, Slattery RM. Transgenic rescue implicates beta2-microglobulin as a diabetes susceptibility gene in nonobese diabetic (NOD) mice. Proc N atl A cad Sa U SA 2001. 98:11533-11538.

29. Kissler S, Stern P, T akahashi K, Hunter K, Peterson LB, Wicker LS. In vivo R N A interference demonstrates a role for $\mathrm{N}$ ramp1 in modifying susceptibility to type 1 diabetes. $N$ at $\mathrm{G}$ enet 2006. 38:479-483.

30. Gerold KD, Z heng $\mathbf{P}, \mathbf{R}$ ainbow DB, Zernecke $A$, Wicker LS, Kissler S. The soluble CT LA-4 splice variant protects from type 1 diabetes and potentiates regulatory $T$ cell function. D iabetes 2011. 60:1955-1963.

31. Z heng $\mathbf{P}, \mathbf{K i s s l e r} \mathbf{S}$. PTPN 22 Silencing in the NOD M odel Indicates the Type 1 Diabetes-Associated Allele Is $\mathrm{N}$ ot a Loss of- Function Variant. D iabetes 2012. In press.

32. Nagafuchi S, Katsuta H, Kogawa K, Akashi T, Kondo S, Sakai Y, T sukiyama T, Kitamura D, Niho $\mathbf{Y}$, Watanabe T. Establishment of an embryonic stem (ES) cell line derived from a non-obese diabetic (N OD) mouse: in vivo differentiation into lymphocytes and potential for germ line transmission. FE BS Lett 1999. 455:101-104.

33. Arai S, Minjares C, Nagafuchi S, Miyazaki T. Improved experimental procedures for achieving efficient germ line transmission of nonobese diabetic (N OD)-derived embryonic stem cells. Exp D iabesity R es 2004. 5:219-226.

34. Chen J, Reifsnyder PC, Scheuplein F, Schott WH, Mileikovsky M, Soodeen-Karamath S, Nagy A, Dosch MH, Ellis J, Koch-Nolte F, Leiter EH. "Agouti NOD": identification of a CBA-derived Idd locus on Chromosome 7 and its use for chimera production with N OD embryonic stem cells. M amm G enome 2005. 16:775783.

35. Brook FA, Evans EP, Lord CJ, Lyons PA, Rainbow DB, Howlett SK, Wicker LS, Todd JA, Gardner RL. The derivation of highly germline-competent embryonic stem cells containing N O D-derived genome. D iabetes 2003.

\section{2:205-208}

36. Ying Q L, Wray J, Nichols J, Batlle-Morera L, Doble B, Woodgett J, Cohen P, Smith A. The ground state of embryonic stem cell self-renewal. N ature 2008. 453:519-523.

37. O hta H, Ohinata $Y$, Ikawa M, Morioka $Y$, Sakaide $Y$, Saitou M, Kanagawa O, Wakayama T. M ale germline and embryonic stem cell lines from NOD mice: efficient derivation of $\mathrm{GS}$ cells from a nonpermissive strain for $\mathrm{ES}$ cell derivation. Biol R eprod 2009. 81:1147-1153.

38. Yang W, Wei W, Shi $C, Z$ hu J, Ying W, Shen $Y, Y e$ $X$, Fang L, Duo S, Che J, et al. Pluripotin combined with leukemia inhibitory factor greatly promotes the derivation of embryonic stem cell lines from refractory strains. Stem C ells 2009. 27:383-389.

39. Hanna J, Markoulaki S, Mitalipova M, Cheng AW, C assady JP, Staerk J, Carey BW, Lengner CJ, Foreman R, Love J, et al. M etastable pluripotent states in N O D-mouse-derived ESC s. C ell Stem C ell 2009. 4:513-524.

40. Nichols J, Jones K, Phillips JM, Newland SA, R oode M, Mansfield W, Smith A, Cooke A. Validated germline-competent embryonic stem cell lines from nonobese diabetic mice. N at M ed 2009. 15:814-818.

41. Morgan MA, Muller PS, Mould A, Newland SA, Nichols J, Robertson EJ, Cooke A, Bikoff EK. The $\mathrm{N}$ onconventional M HC class II molecule DM governs diabetes susceptibility in NOD mice. PLOS O ne 2013. 8:e56738.

42. Kamanaka M, Rainbow D, Schuster-Gossler K, Eynon EE, Chervonsky AV, Wicker LS, Flavell RA. A mino acid polymorphisms altering the glycosylation of IL-2 do not protect from type 1 diabetes in the N OD mouse. P roc $N$ atl A cad Sci U SA 2009. 106:11236-11240.

43. Liew CG, Andrews PW. Stem cell therapy to treat diabetes mellitus.. R ev D iabet Stud 2008. 5(4):203-219.

44. Wallis RH, Wang K, Marandi L, Hsieh E, Ning T, Chao GY, Sarmiento J, Paterson AD, Poussier P. Type 1 diabetes in the $B B$ rat: a polygenic disease. $D$ iabetes 2009. 58:1007-1017.

45. Geurts AM, Cost GJ, Freyvert $Y$, Z eitler B, Miller JC, Choi VM, Jenkins SS, Wood A, Cui $X$, Meng $X$, et al. Knockout rats via embryo microinjection of zinc-finger nucleases. Sience 2009. 325:433.

46. Mashimo T, Takizawa A, Voigt B, Yoshimi K, Hiai H, Kuramoto T, Serikawa T. Generation of knockout rats with $X$-linked severe combined immunodeficiency $(X$ SCID) using zinc-finger nucleases. PL OS 0 ne 2010. 5:e8870.

47. Cui $\mathbf{X}$, Ji $\mathbf{D}$, Fisher DA, Wu $\mathbf{Y}$, Briner DM, Weinstein EJ. Targeted integration in rat and mouse embryos with zinc-finger nucleases. N at Biotechnol 2011. 29:6467.

48. Tesson L, Usal C, Ménoret S, Leung E, Niles BJ, Remy S, Santiago $Y$, Vincent Al, Meng X, Z hang $L$, et al. K nockout rats generated by embryo microinjection of TALEN s. N at Biotechnol 2011. 29:695-696.

49. Meyer M, Ortiz $\mathbf{O}$, Hrabe de Angelis $\mathbf{M}$, Wurst $\mathbf{W}$, Kühn R. Modeling disease mutations by gene targeting in one-cell mouse embryos. Proc N atl A cad Sc USA 2012. 109:9354-9359.

50. Wang J, Friedman G, Doyon Y, Wang NS, Li CJ, Miller JC, Hua KL, Yan J, Babiarz JE, Gregory PD, Holmes MC. Targeted gene addition to a predetermined site in the human genome using a ZFN -based nicking enzyme. $\mathrm{G}$ enome R es 2012. 22:1316-1326. 
51. Cohen AJ, McGill PD, Rossetti RG, Guberski DL, Like AA. Glomerulopathy in spontaneously diabetic rat. Impact of glycemic control. D iabetes 1987. 36:944-951.

52. Many MC, Maniratunga S, Denef JF. The non-obese diabetic (NOD) mouse: an animal model for autoimmune thyroiditis. Exp Clin Endocrinol D iabetes 1996. 104(Suppl 3): $17-20$

53. Allen EM, Appel MC, Braverman LE. The effect of iodide ingestion on the development of spontaneous lymphocytic thyroiditis in the diabetes-prone $\mathrm{BB} / \mathrm{W}$ rat. Endocrinology 1986. 118:1977-1981.

54. Thompson C, Jacobsen H, Pomeranz Krummel D, Nagai K, Cooke A. N on-depleting anti-CD 4 antibody not only prevents onset but resolves sialadenitis in NOD mice. A utoimmunity 2004. 37:549-554.

55. Bernard C C, Johns T G, Slavin A, Ichikawa M, Ewing C, Liu J, Bettadapura J. M yelin oligodendrocyte glycoprotein: a novel candidate autoantigen in multiple sclerosis. M ol M ed (Berl) 1997. 75:77-88.

56. Smith RJ, Sly LM. Type II collagen-induced arthritis in the diabetic-resistant biobreeding rat: inflammatory and histopathological features of joint pathology and effects of antiinflammatory and antirheumatic drugs on this chronic arthritic process. J P harmacol Exp T her 1996. 277:1801-1813.

57. Cha S, Nagashima H, Brown VB, Peck AB, Humphreys-Beher MG. Two NOD Idd-associated intervals contribute synergistically to the development of autoimmune exocrinopathy (Sjögren's syndrome) on a healthy murine background. A rthritis R heum 2002. 46:1390-1398.

58. Baker D, R osenwasser OA, O'Neill JK, Turk JL. Genetic analysis of experimental allergic encephalomyelitis in mice. J I mmunol 1995. 155:4046-4051.

59. del $\mathbf{R}$ io $\mathbf{R}$, Noubade $\mathbf{R}$, Subramanian $\mathbf{M}$, Saligrama $\mathbf{M}$, Diehl S, R incon $\mathbf{M}$, Teuscher $\mathbf{C}$. SN Ps upstream of the minimal promoter control IL-2 expression and are candidates for the autoimmune disease-susceptibility locus A od2/Idd3/Eae3. G enes and Immunity 2008. 9:115-121.

60. Testa G, Z hang Y, Vintersten K, Benes V, Pijnappel WW, Chambers I, Smith AJ, Smith AG, Stewart AF. Engineering the mouse genome with bacterial artificial chromosomes to create multipurpose alleles. $\mathrm{N}$ at Biotechnol 2003. 21:443-447. 